\title{
Highest-energy cosmic rays from Fermi-degenerate relic neutrinos consistent with Super-Kamiokande results
}

\author{
Graciela Gelmini and Alexander Kusenko \\ Department of Physics and Astronomy, UCLA, Los Angeles, CA 90095-1547
}

(February, 1999)

\begin{abstract}
Relic neutrinos with mass $0.07_{-0.04}^{+0.02} \mathrm{eV}$, in the range consistent with Super-Kamiokande data, can explain the cosmic rays with energies in excess of the Greisen-Zatsepin-Kuzmin cutoff. The spectrum of ultra-high energy cosmic rays produced in this fashion has some distinctive features that may help identify their origin. Our mechanism does not require but is consistent with a neutrino density high enough to be a new kind of hot dark matter.
\end{abstract}

PACS numbers: 98.70.Sa, 95.85.Ry, 14.60.Pq, 95.35.+d $\quad$ UCLA/99/TEP/6

The observation of atmospheric neutrino oscillations at Super-Kamiokande has provided a strong evidence that at least one of the neutrino species has mass greater than $m_{S K}=\sqrt{\delta m^{2}}=0.07_{-0.04}^{+0.02} \mathrm{eV}$. It seems plausible that at least one of the neutrino masses is actually in this range, as would be the case if the neutrino masses were hierarchical. Of course, an alternative possibility, that some neutrino masses are nearly degenerate and larger than $\sqrt{\delta m^{2}}$, is also consistent with the current data. In this paper we will concentrate on the former case.

If one of the lepton asymmetries of the Universe $L_{i}=$ $\left(n_{\nu_{i}}-n_{\bar{\nu}_{i}}\right) / s$ is of order one [1], the neutrinos with masses $m_{S K}$ can make a significant contribution to the energy content of the Universe [5]. (Here and below, $n_{x}$ denotes the number density of the $x$-species, and $s=1.80 g_{s *} n_{\gamma}$ is the entropy density.) This possibility, frequently discounted in contemporary cosmology, can arise naturally if the Universe underwent an AffleckDine baryo- and leptogenesis [6] at the end of a relatively low-scale inflation. Lepton asymmetries can also be generated in neutrino oscillations [7] after the electroweak phase transition, but we do not know whether an asymmetry of order one can arise in this fashion.

We will show that ultra-high energy cosmic rays with interesting new features can be produced in the presence of such background neutrinos.

The cosmic rays with energies beyond the GreisenZatsepin-Kuzmin (GZK) cutoff [8] present a challenging outstanding puzzle in astroparticle physics and cosmology [9.10]. The protons with energies above $5 \times 10^{19} \mathrm{eV}$ could not reach Earth from a distance beyond $50-100$ Mpc 111 because they scatter off the cosmic microwave background photons with a resonant photoproduction of pions: $p \gamma \rightarrow \Delta^{*} \rightarrow N \pi$. The mean free path for this reaction is only $6 \mathrm{Mpc}$. The photons of comparable energies pair-produce electrons and positrons on the radio background and, likewise, cannot reach Earth from beyond 10 to $40 \mathrm{Mpc}$ [12]. This creates a problem because the closest astrophysical objects that could produce such energetic particles, active galactic nuclei (AGN), are at least hundreds of megaparsecs away.

Several solutions have been proposed for the origin of these ultra-high energy cosmic rays (UHECR). For example, they could be produced in the decay of some ubiquitous hypothetical heavy particles 13], topological defects 14], or light neutral supersymmetric hadrons [15]; or they could also hint at exotic interactions [16]. A more conservative and economical scenario involves relic neutrinos. It has been suggested [17] that distant AGN's can produce high-energy neutrinos whose annihilation on the relic neutrinos in the galactic halo at $\sqrt{s} \approx M_{z}$ can produce the protons with energies above the GZK cutoff. In the absence of lepton asymmetry, the background neutrino density would not be sufficient to generate the necessary flux of UHECR if it were not for the clustering of neutrinos in the galactic halo. The latter helps. However, the required total energy carried by the high-energy neutrinos is uncomfortably close to the total luminosity of the Universe 18.

If neutrinos with mass $m_{S K}$ carry a large lepton asymmetry, the above scenario is aided in several ways. First, the density $n_{\nu}$ of the Fermi-degenerate light neutrinos should be much higher than that considered in Ref. [17]. Therefore, the neutrino annihilations in the entire volume $\sim(50 \mathrm{Mpc})^{3}$ contribute to the observed UHECR. Second, the probability for the neutrinos emitted at distances of the order of the inverse Hubble constant, $\sim H^{-1}$, to decay within $50 \mathrm{Mpc}$ of the observer is maximal when the mean free path $\lambda=1 /\left(\sigma_{\text {ann }} n_{\nu}\right)$ is comparable to $H^{-1}$. We will see that this condition, $\lambda \sim H^{-1}$, is satisfied automatically for $m_{\nu}$ measured by Super-Kamiokande if $\Omega_{\nu} h^{2} \sim 0.01$. As a result of an increased background neutrino density and the increase in annihilation probability in our neighborhood, the total energy of the energetic neutrinos is much less than the total luminosity of the Universe, in contrast to Refs. [17, 18]. Finally, the predicted spectrum of the UHECR peaks at $10^{20-21} \mathrm{eV}$ and has a cutoff at about $10^{23} \mathrm{eV}$, hence, creating an observable feature that could help distinguish this mechanism from some others. 
As long as the chemical potential of the degenerate neutrinos $\mu$ is smaller than the neutrino mass, the neutrinos are non-relativistic. Therefore, the neutrinos with mass $m_{S K}$ are non-relativistic at present if the degeneracy parameter $\xi=\mu / T<100$. We will not come close to this upper bound on $\xi$. Therefore, the energy density is $\rho_{i}=m_{\nu_{i}} n_{\nu_{i}}$ and

$$
\eta_{\nu}=\frac{n_{\nu}}{n_{\gamma}}=3.6\left(\frac{0.07 \mathrm{eV}}{m_{\nu}}\right)\left(\frac{\Omega_{\nu} h^{2}}{0.01}\right),
$$

where $\Omega_{\nu} \equiv \rho_{i} / \rho_{c}$.

These neutrinos decouple while they are still relativistic because the decoupling temperature $T_{d}$ increases with $\xi$ [19], so $T_{d}>1 \mathrm{MeV}$. Therefore, the present value of $\xi$ is determined by the following relation valid for relativistic species:

$$
\eta=\frac{1}{12 \zeta(3)}\left(\frac{T_{\nu}}{T_{\gamma}}\right)^{3}\left[\pi^{2} \xi+\xi^{3}\right]=0.0252\left(9.87 \xi+\xi^{3}\right) .
$$

Here we used $\zeta(3)=1.202$ and $\left(T_{\nu} / T_{\gamma}\right)^{3}=4 / 11$. This relation is valid as long as $T_{d}$ is lower than the muon mass, which translates into the upper bound $\xi<12$ 19. From equation (2), $\eta=3.6$, as in equation (11), corresponds to $\xi=4.6$.

The UHECR are dominated by the resonant neutrino annihilations with $\sqrt{s} \approx M_{Z}$, which corresponds to the incoming neutrino energy $E_{\nu}=M_{z}^{2} / 2 m_{\nu}=0.57 \times$ $10^{23} \mathrm{eV}\left(m_{\nu} / 0.07 \mathrm{eV}\right)$. The annihilation cross section is $\sigma_{\text {ann }}=4 \pi G_{F} / \sqrt{2}$. The mean free path for a neutrino is, therefore,

$$
\begin{aligned}
\lambda=\frac{1}{\sigma_{\mathrm{ann}} n_{\nu}} & =5.3\left(\frac{412 \mathrm{~cm}^{-3}}{n_{\nu}}\right) \times 10^{28} \mathrm{~cm} \\
& =\frac{3.9}{\eta_{\nu}}\left(\frac{0.65}{h}\right) H^{-1} .
\end{aligned}
$$

The atmospheric neutrino oscillations observed at Super-Kamiokande imply that a muon neutrino has a large, order one, mixing with either $\nu_{\tau}$ or a sterile neutrino. The mass eigenstate that makes up the Fermidegenerate relic background with mass $m_{S K}$ must, therefore, have a muon neutrino component that is not small. The astrophysical sources are expected to produce highenergy muon neutrinos from the decays of pions. Since both the background neutrinos and the high-energy neutrinos from astrophysical sources must have a large muon component, there is no significant suppression of the annihilation cross section due to mixing angles.

The probability of the neutrino annihilation is maximized when $\eta_{\nu} \simeq 4$, at which point a fraction $r=$ $(50 \mathrm{Mpc} / 2.7 \lambda) \approx 5.5 \times 10^{-3}$ of all neutrinos with energies near the $Z$ resonance annihilate within $50 \mathrm{Mpc}$ of the observer. According to the data pertaining to the momentum distribution of $Z$ decay products 20], these neutrino annihilations yield hadrons with an average energy

$$
E_{p} \sim 0.025 E_{\nu} \approx 1.4\left(\frac{0.07 \mathrm{eV}}{m_{\nu}}\right) \times 10^{21} \mathrm{eV}
$$

and photons (from the decays of $\pi^{0}$ ) with a lower average energy,

$$
E_{\gamma} \sim 0.0035 E_{\nu} \approx 2.0\left(\frac{0.07 \mathrm{eV}}{m_{\nu}}\right) \times 10^{20} \mathrm{eV} .
$$

$Z$ decays produce on average 2 nucleons and about 9.5 $\pi^{0}$ 's, which decay into 19 photons [20]. Even though the photons that reach Earth originate in a smaller volume $(10-40 \mathrm{Mpc})^{3}$ than protons $(50-100 \mathrm{Mpc})^{3}$, both components should contribute to the UHECR because the multiplicity of photons is about 10 times greater than that of protons. It may be possible, given enough statistics of UHECR, to resolve the two peaks in the distribution of cosmic rays: one at lower mean energy, due to photons, and another, at higher energies, due to protons.

The total energy per unit volume in neutrinos with energies above $10^{19} \mathrm{eV}$ in our case is lower than that in Refs. 17, 18] by a factor $21\left(\eta_{\nu} / 4\right)\left(m_{\nu} / 0.07 \mathrm{eV}\right)$. The difference with $E\left(>10^{19} \mathrm{eV}\right)$ in equation (5) of Ref. 18] is the increased value of the background neutrino density, $m_{\nu}=m_{S K}$, and $N_{C R} \sim 30$. This means the total power generated in high-energy neutrinos $\mathcal{E}_{\nu} \sim$ $0.5\left(4 / \eta_{\nu}\right)\left(0.07 \mathrm{eV} / m_{\nu}\right) 10^{48} \mathrm{erg} \mathrm{Mpc}^{-1} \mathrm{yr}^{-1}$ is well below the luminosity of the Universe.

Bounds on the neutrino degeneracy come from nucleosynthesis [19.21], as well as structure formation in the Universe 22]. A combination of both yields $-0.06 \lesssim$ $\xi_{\nu_{e}} \lesssim 1.1,\left|\xi_{\nu_{\mu, \tau}}\right| \lesssim 6.919$. In addition, in models with large neutrino degeneracy, the baryon density of the Universe can be higher than in conventional nucleosynthesis, so a larger fraction of the dark matter can be baryonic. These bounds are based on the requirement that neutrinos not interfere with galaxy formation. However, it has been shown recently [23] that a relativistic degenerate neutrino may actually help the formation of structure in the Universe. This neutrino may be the addition that 'standard' cold dark matter (CDM) models need to account well for all present data on structure in the Universe. In fact, a CDM model with a relativistic relic neutrino background with $\xi \sim 3.4$ [23] provides a good fit to all the data on large-scale structure and anisotropy of the cosmic microwave background radiation. These analyses do not fully apply to our case because the neutrinos with mass $m_{S K}$ are non-relativistic at present. Our scenario is consistent with large values of $\Omega_{\nu} h^{2}$ that can make the relic degenerate neutrinos an important hot dark matter component. Its effects on structure formation and the anisotropy of CMBR need to be studied.

Finally, we would like to address the issue of how the large lepton asymmetry could be generated in the early 
Universe, while the baryon asymmetry remains small. The coherent Affleck-Dine condensate could have evolved differently along the flat directions carrying the baryon $(B)$ and the lepton $(L)$ numbers. The corresponding supersymmetry-breaking terms and higher-dimension operators [24] depend on the type of a flat direction and need not be the same for the directions with $B \neq 0$ and $L \neq 0$. If the electroweak symmetry was never restored after inflation, either because the reheat temperature was low, or because the lepton asymmetry was high [2, 1 , the baryon and lepton asymmetries of the Universe may differ by many orders of magnitude. A cold dark matter component, called for by the need to form structure, could also arise naturally in the low-scale Affleck-Dine scenario [25]. In this paper we do not assume any particular cosmological scenario for generating the relic neutrinos. However, it is reassuring that an economical selfconsistent cosmological model outlined above can simultaneously produce cold and hot dark matter, the latter being the light, Fermi-degenerate neutrinos that carry a lepton asymmetry of order one.

To summarize, we have shown that a cosmic background of Fermi-degenerate neutrinos with masses inferred from the Super-Kamiokande data can explain the ultra-high energy cosmic rays above the GZK cutoff. Our mechanism does not require but is consistent with a neutrino density high enough to be a new kind of hot dark matter. The mechanism predicts ultra-high energy cosmic rays from both protons, peaked at energies $10^{22} \mathrm{eV}$, and photons, peaked at $10^{20} \mathrm{eV}$. Another prediction is a new cutoff at $M_{Z}^{2} / 2 m_{\nu}=0.57 \times 10^{23} \mathrm{eV}\left(m_{\nu} / 0.07 \mathrm{eV}\right)$.

This work was supported in part by the US Department of Energy grant DE-FG03-91ER40662, Task C.

[1] A. D. Dolgov and D. P. Kirilova, J. Moscow Phys. Soc. 1, 217 (1991); A. D. Dolgov, Phys. Rep. 222, 309 (1992).

[2] A. Casas, W. Y. Cheng, and G. Gelmini, Nucl. Phys. B538, 297 (1999).

[3] J. A. Harvey and E. W. Kolb, Phys. Rev. D24, 2090 (1981); J. Liu and G. Segrè, Phys. Rev. D48, 4609 (1993); B. Bajc, A. Riotto, and G. Senjanović, Phys. Rev. Lett. 81, 1355 (1998).

[4] A. Linde, Phys. Rev. D14, 3345 (1976); P. Langacker, G. Segrè, and S. Soni, Phys. Rev. D26, 3425 (1982); J. Liu and G. Segrè, Phys. Lett. B338, 259 (1994).

[5] P. Pal and K. Kar, hep-ph/9809410.

[6] I. Affleck and M. Dine, Nucl. Phys. B249, 361 (1985).

[7] R. Foot, M. J. Thompson, and R. R. Volkas, Phys. Rev. D53, 5349 (1996); X. Shi, Phys. Rev. D54, 2753 (1996).

[8] K. Greisen, Phys. Rev. Lett. 16, 748 (1966); G. T. Zatsepin and V. A. Kuzmin, Pisma Zh. Eksp. Teor. Fiz. 4, 114 (1966).
[9] D. J. Bird et al., Phys. Rev. Lett. 71, 3401 (1993); Astrophys. J. 424, 491 (1994).

[10] S. Yoshida et al., Astropart. Phys. 3, 151 (1995).

[11] S. Yoshida and M. Teshima, Prog. Theor. Phys. 89, 833 (1993); F. A. Aharonian and J. W. Cronin, Phys. Rev. D50, 1892 (1994); J. W. Elbert and P. Sommers, Astrophys. J. 441, 151 (1995);

[12] F. Halzen, R. A. Vazquez, T. Stanev, and V. P. Vankov, Astropart. Phys., 3, 151 (1995).

[13] V. Berezinsky, M. Kachelriess, and A. Vilenkin, Phys. Rev. Lett. 79, 4302 (1997); V. A. Kuzmin and V. A. Rubakov, Phys. Atom. Nucl. 61, 1028 (1998) [Yad. Fiz. 61, 1122 (1998)]; M. Birkel and S. Sarkar, Astropart. Phys. 9, 297 (1998); V. Kuzmin and I. Tkachev, JETP Lett. 68, 271 (1998); K. Benakli, J. Ellis, and D. V. Nanopoulos, Phys. Rev. D59, 047301 (1999).

[14] C. T. Hill, D. N. Schramm, and T. P. Walker, Phys. Rev. D36, 1007 (1987); G. Sigl, D. N. Schramm and P. Bhattacharjee, Astropart. Phys. 2, 401 (1994); V. Berezinsky, X. Martin, and A. Vilenkin, Phys.Rev. D56, 2024 (1997).

[15] D. J. Chung, G. R. Farrar, and E. W. Kolb, Phys. Rev. D57, 4606 (1998); G. R. Farrar and P. Biermann, Phys. Rev. Lett. 81, 3579 (1998); I. F. Albuquerque, G. R. Farrar, and E. W. Kolb, Phys. Rev. D59, 015021 (1999).

[16] G. Domokos and S. Kovesi-Domokos, Phys. Rev. Lett. 82, 1366 (1999).

[17] D. Fargion, B. Mele, and A. Salis, astro-ph/970029; T. Weiler, hep-ph/9710431.

[18] E. Waxman, astro-ph/9804023.

[19] H. Kang and G. Steigman, Nucl. Phys. B372, 494 (1992).

[20] Particle Data Group, Eur. Phys. J. C3, 201-202 (1998).

[21] R. V. Wagoner, W. A. Fowler and F. Hoyle, Ap. J. 148, 3 (1967); A. Yahil and G. Beaudet, Ap. J. 206, 26 (1976); Y. David and H. Reeves, Physical Cosmology, ed. by R. Balian, J. Audouze, and D. N. Schramm, NorthHolland, Amsterdam, 1980; J. N. Fry and C. J. Hogan, Phys. Rev. Lett. 49, 1783 (1982); R. J. Scherrer, Mon. Not. Roy. Astron. Soc. 205, 683 (1983); N. Terazawa and K. Sato, Ap. J. 294, 9 (1985); K. Olive, D. N. Schramm, D. Thomas and T. Walker, Phys. Lett. B265, 239 (1991); G. Starkman Phys. Rev. D45, 476 (1992).

[22] K. Freese, E. W. Kolb, and M. S. Turner, Phys. Rev. D27, 1689 (1983).

[23] J. Adams and S. Sarkar, talk presented at the ICTP Workshop on physics of relic neutrinos, Trieste, Italy, 1998.

[24] M. Dine, L. Randall, and S. Thomas, Phys. Rev. Lett. 75, 398 (1995); Nucl. Phys. B458, 291 (1996); J. A. Casas and G. B. Gelmini, Phys. Lett. B410, 3 (1997); B. A. Campbell, M. K. Gaillard, H. Murayama, and K. A. Olive, Nucl. Phys. B538, 351 (1999); M. Axenides, E. G. Floratos, G. K. Leontaris, and N. D. Tracas, hep-ph/9811371.

[25] A. Kusenko and M. Shaposhnikov, Phys. Lett. B418, 46 (1998); K. Enqvist and J. McDonald, Phys. Lett. B 425, 309 (1998). 